\title{
Bacteroidales species are a reservoir of phase-variable antibiotic resistance genes in the human gut microbiome
}

Wei Yan ${ }^{a}, A$. Brantley Hall ${ }^{b, c}$, and Xiangfang Jiang ${ }^{a}$

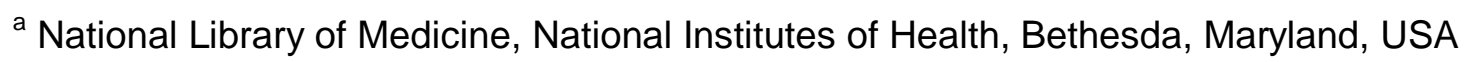

${ }^{\mathrm{b}}$ Department of Cell Biology and Molecular Genetics, University of Maryland, College Park, Maryland, USA

${ }^{\mathrm{C}}$ Center for Bioinformatics and Computational Biology, University of Maryland, College Park, Maryland, USA

Address correspondence to Xiaofang Jiang: xiaofang.jiang@nih.gov 


\section{ABSTRACT}

Phase-variable antibiotic resistance genes (ARGs) can mitigate the fitness cost of maintaining ARGs in the absence of antibiotics and could potentially prolong the persistence of ARGs in bacterial populations. However, the origin, prevalence, and distribution of phase-variable ARGs remains poorly understood. Here, we sought to assess the threat posed by phase-variable ARGs by systematically searching for phase-variable ARGs in the human gut microbiome and examining their origin, prevalence, and distribution. Through metagenomic assembly of 2227 human gut metagenomes and genomic analysis of the Unified Human Gastrointestinal Genome (UHGG) collection, we identified phase-variable ARGs and categorized them into three classes based on the invertase regulating phase variation. In the human gut microbiome, phase-variable ARGs are commonly and exclusively distributed in Bacteroidales species. Through genomic analysis, we observed that phase-variable ARGs have convergently originated from ARG insertions into phase-variable capsule polysaccharide biosynthesis (CPS) loci at least three times. Moreover, all identified phase-variable ARGs are located within integrative conjugative elements (ICEs). Therefore, horizontal transfer via ICEs could explain the wide taxonomic distribution of phase-variable ARGs. Overall, these findings reveal that phase-variable CPS loci in Bacteroidales species are an important hotspot for the emergence of clinically-relevant phase-variable ARGs. 


\section{INTRODUCTION}

The proliferation of antibiotic resistance genes (ARGs) has compromised antibiotic treatment for bacteria infections (1). The human gut microbiome is an important reservoir of ARGs (2-4) and the spread of ARGs from gut microbes to pathogens has been documented (5). Therefore, ARGs in the human gut microbiome pose a growing threat to human health.

Often, bacteria carrying ARGs are outcompeted by susceptible strains due to the costs associated with the maintenance and expression of the ARGs (6-8). Though it is costly, bacteria can ameliorate the fitness costs of maintaining ARGs through different strategies (9), such as no-cost, low-cost or gain of fitness mutations $(10,11)$, compensatory mutations at a second site (12-14), or genetic co-selection of resistance genes in genetic linkage $(15,16)$. Phase-variable ARGs, which were only recently reported (17), are a newly-identified mechanism for antibiotic resistant bacteria to mitigate the fitness cost of encoding ARGs.

Phase variation refers to a reversible change that generates phenotypic variation which helps bacteria adapt to rapidly changing environments $(18,19)$. Phase variation often manifests through reversible inversion of DNA regions containing promoters such that in one orientation a downstream gene is expressed while in the alternate orientation, the downstream gene is not expressed (17). Such DNA inversions are generally mediated by invertases, which recognize inverted repeats flanking the invertible region and catalyze the reversible inversion (20-22). Phase-variable genes often regulate characteristics important for bacterial colonization and virulence including fimbriae $(23,24)$, flagella $(25)$, and capsular polysaccharides (CPS) $(26,27)$.

Recent advances in computational methods have contributed to the effective identification of the intergenic invertible DNA regions in microbial genomes $(17,28)$. ARGs were found to be regulated by invertible promoters in certain human gut bacteria (17). Resistant bacteria with phase-variable ARGs could maintain these ARGs for a longer period of time in the microbial community, by switching the expression of ARGs to the OFF orientation in the absence of antibiotics to ameliorate the fitness cost (17). The emergence of phase-variable ARGs increases the burden for humans to combat antibiotic resistance and the spread of phase-variable ARGs to pathogens poses an increasing threat to human health. Therefore, we need a better understanding of the evolution of phase-variable ARGs as well as their taxonomic and geographic distribution to combat them.

Here, we took advantage of the considerable amount of human gut metagenomic data generated during the last decade to examine the origin, evolution and prevalence of phase- 
variable ARGs on a large scale. We systematically searched for phase-variable ARGs through metagenomic assembly of 2227 human gut metagenomes and the Unified Human Gastrointestinal Genome (UHGG) collection of human gut genomes. We found that phasevariable ARGs were commonly distributed in Bacteroidales species. Genomic analysis showed that phase-variable ARGs have been convergently derived from ARG insertions into phasevariable CPS loci. Notably, the identified phase-variable ARGs were found to have been mobilized through ICEs and have been widely geographically distributed. Our results reveal the prominent role of phase-variable CPS loci in Bacteroidales species in providing hotspots for the emergence of clinically-relevant phase-variable ARGs.

\section{RESULTS}

\section{Identification and classification of phase-variable antibiotic resistance genes}

To expand the known repertoire of phase-variable ARGs, we searched for phase-variable ARGs from publically available human gut metagenomic datasets, comprising a total of 2227 samples, using the tool PhaseFinder (Table S1). Briefly, we assembled the metagenomic data then searched for phase-variable regions with PhaseFinder by aligning the unassembled reads back to the metagenomic assemblies. Then, phase-variable ARGs were identified by scanning regions downstream of an identified phase-variable region for ARGs. In total, this search uncovered 61 contigs containing phase-variable ARGs. In all phase-variable ARGs identified, the invertible region was located immediately downstream of a gene encoding an invertase. We identified putative promoter sequences in the invertible region of all 61 phase-variable regions. Therefore, we concluded that these 61 ARGs are likely regulated by invertible promoters.

To analyze the origin and evolution of phase-variable ARGs, we constructed a phylogenetic tree based on the nucleotide sequence of the invertases. We grouped the invertible promoters regulating ARGs (IP-ARGs) into three distinct classes, denoted IP-ARG-1, IP-ARG-2, and IPARG-3 (Figure 1, Table S2).

To further characterize these three classes of phase-variable ARGs, we analyzed the motifs of inverted repeats and invertible promoters as well as the ARGs regulated by the invertible regions. The invertible promoter motifs varied among classes but were nearly identical within the same class (Figure 1). The inverted repeat motifs were found to be similar across different classes (Figure 1). Interestingly, we found two invertible regions located immediately downstream of the invertase gene in class IP-ARG-2 (Figure 1). The two invertible regions were 
located adjacent to each other and the inverted repeats were nearly identical in these two regions. This suggested that these two invertible regions could be regulated by the same upstream invertase gene. However, the invertible promoters were different from each other (Figure 1, Table S2). This suggested that these two invertible regions might generate more phenotypic variations than only one invertible region does. The ARGs regulated by invertible promoters varied among classes (Figure 1, Table S2). Most invertible promoters of class IPARG-1 regulated the $c m e A B C$ operon, ermG gene, or both, while some also regulated other ARGs, including mel, msrE, tetQ, or ermF. These ARGs could confer resistance to diverse antibiotics, including cephalosporin, fluoroquinolone, fusidic acid, lincosamide, macrolides, oxazolidinone, phenicol, pleuromutilin, streptogramin, and tetracycline. The ermB gene and the ermF gene, conferring resistance to streptogramin, macrolides, and lincosamides, were found to be regulated by class IP-ARG-2 and class IP-ARG-3, respectively. In addition, class IP-ARG-2 was also found to regulate an ant(9) gene homolog which confers resistance to aminoglycosides.

\section{Taxonomic distribution of phase-variable ARGs}

To examine the geographic and taxonomic distribution of phase-variable ARGs, we searched genomes from the Unified Human Gastrointestinal Genome (UHGG) collection (29) to detect nearly identical (> 99\% identity) invertase genes. The invertase genes mediating ARGs were grouped into the corresponding class of phase-variable ARGs (Table S3). Phase-variable ARGs were observed in multiple countries, but the geographic prevalence varied across classes (Table 1, Table S2, and Table S3). Phase-variable ARGs from classes IP-ARG-1 and IP-ARG-2 were identified in metagenomes from 17 and 7 countries, respectively, spanning three continents (Asia, Europe, and North America). Phase-variable ARGs from class IP-ARG-3 were only observed in the metagenomes from Denmark, which might be due to the limited sampling of publically-available metagenomic data. The results reveal that species harboring phasevariable ARGs are widely geographically distributed.

Phase-variable ARGs were found to be commonly and exclusively distributed in Bacteroidales species (Table 1, Table S2, and Table S3). Phase-variable ARGs belonging to class IP-ARG-1 were observed in 28 Bacteroidales species from the families Bacteroidaceae, Barnesiellaceae, Coprobacteraceae, and Tannerellaceae. Phase-variable ARGs belonging to class IP-ARG-2 could be identified in 6 species from Bacteroidaceae that belonged to the order Bacteroidales. Class IP-ARG-3 was found in two species, Phocaeicola dorei and a novel species RC9 sp000434935, which also belongs to the Bacteroidales order. These suggested that phase- 
variable ARGs are restricted in Bacteroidales. The wide taxonomic distribution combined with the sparse occurrence of these phase-variable ARGs is not consistent with vertical transmission, suggesting that phase-variable ARGs could be horizontally transferred by mobile genetic elements (MGEs).

\section{All three classes of phase-variable ARGs are located within integrative conjugative elements}

To determine whether the phase-variable ARGs were on MGEs, we searched the invertases and invertible regions regulating phase-variable ARGs as well as the flanking sequences against ImmeDB (30) and the ICEberg database (31). We found that all three classes of phasevariable ARGs are located within integrative conjugative elements (ICEs) (Flgure 2). The majority of phase-variable ARGs from class IP-ARG-1 were identified within ICEs related to ICE26 in the ImmeDB (Figure 2a). Phase-variable ARGs from class IP-ARG-1 were also found within another novel ICE (Figure 2a). This suggested that the progenitor of IP-ARG-1 might have emerged before subsequently inserting into multiple ICEs. Phase-variable ARGs from class IP-ARG-2 were detected in ICEs related to ICE14 in ImmeDB (Figure 2b). A phasevariable ARG from class IP-ARG-3 contained within a 50-kb sequence fragment most closely matches ICE34 in ImmeDB (Figure 2c). The fact that all three classes of phase-variable ARGs are located within ICEs is medically relevant because ICEs encode the necessary machinery to horizontally transfer between species mobilizing any ARGs that they acquire.

Phase-variable ARGs were frequently observed to be included in a highly variable region that was located immediately downstream of the invertible regions. The highly varied regions contained genes that were not necessary for the ICE replication and transfer, but often important for conferring selective and adaptive advantages for hosts in the changing environments (Figure 2). Most of the variable genes in the highly varied region were ARGs in ICEs containing class IP-ARG-1 (Figure 2a, Table S2), which suggested multiple insertions of ARGs at this region. In ICEs carrying class IP-ARG-2, not only the ARGs but also the CPS genes, even clusters that contained only CPS genes, were located in the regions downstream of the invertases (Figure 2b). In different ICEs with class IP-ARG-3, the highly variable regions contained varied genes or operons, such as ARGs, operons involved in pathogenicity island or T6SS, or integrative and mobilizable elements encoding ARG tetQ (Figure 2c). All these indicated that the loci in such highly variable regions downstream of the invertible promoters were hotspots for the acquisition of elements, including ARGs. 


\section{The invertible regions regulating ARGs appear to originate from those regulating CPS genes independently and convergently}

To better understand the evolution of phase-variable ARGs, we performed comparative analyses on the highly varied regions regulated by invertible promoters. We found that CPS genes, such as wecA gene, or the genes necessary for CPS operon, like upxY gene (32), were frequently located immediately downstream of the invertible promoters and upstream of the identified ARGs (Figure 2a, b, Figure 3). This suggested that the invertible promoters regulating $A R G$ s and those regulating CPS related genes may share the same origin. In addition, the inverted repeats and the invertase amino acids in class IP-ARG-2 shared $100 \%$ and more than $96 \%$ identity with the corresponding sequences of those regulating CPS clusters, respectively (Figure 2b). These results support that the invertible regions regulating ARGs share a common evolutionary origin with invertible regions regulating CPS genes.

Gene context analysis further shows that invertible regions regulating ARGs appeared to be derived from invertible regions originally regulating CPS genes. The phase-variable ARGs were frequently found to be located both upstream and downstream of CPS genes (Figure $2 a, b)$. The genes downstream of the invertible promoters were often partially degenerated, completely degenerated, or fused with the partial sequence of the wecA gene (Figure 2a).

To understand the evolutionary history of different classes of IP-ARGs, a phylogenetic tree was constructed based on the nucleotide sequences of the local invertase genes of known invertible promoters in Bacteroidetes (Figure 3). The result suggests that the evolutionary events that led to the emergence of different classes of phase-variable ARGs occurred independently. Class IPARG-1 might have emerged before being mobilized by ICEs. Class IP-ARG-2 might have emerged as the ARG inserted into the CPS gene cluster loci that had been carried by ICEs (Figure 2a, b). It was difficult to infer the evolutionary history of class IP-ARG-3 because we have identified few sequences closely related to this class. However, the phylogenetic tree revealed that IP-ARG-3 and another two classes were distributed in distinct clades, suggesting that the emergence of class IP-ARG-3 was likely independent of class IP-ARG-1 or IP-ARG-2. Hence, the emergence of different classes of phase-variable ARGs might be the result of convergent evolution and the evolutionary events that led to the emergence of phase-variable ARGs likely occurred independently at least three times. 


\section{DISCUSSION}

In this study, we systematically searched for phase-variable ARGs in human gut microbes and explored the origin, evolution, and prevalence of the phase-variable ARGs. Our analysis revealed that phase-variable ARGs are (a) commonly found in Bacteroidales species, (b) often originate from ARG insertions into phase-variable CPS loci, (c) frequently mobilized by ICEs which may explain their wide taxonomic distribution within the phylum Bacteroidetes and their rapid dissemination, and (d) widely geographically distributed.

Our analysis only identified phase-variable ARGs in Bacteroidales species. There could be several reasons for this observed taxonomic restriction. First, gut Bacteroidales genomes typically contain numerous phase-variable loci. In some Bacteroides species, such as Bacteroides fragilis, there are more than 20 phase-variable loci including up to 7 phase-variable CPS loci $(33,34)$. While other phyla prevalent in the gut, including Proteobacteria, Firmicutes, and Actinobacteria, have phase-variable loci, these loci are far less common and have far fewer examples per genome $(17,35)$. Therefore, ARG insertions into Bacteroidetes phase-variable loci are statistically more likely than insertions into phase-variable loci in other phyla. Furthermore, as abundant members of the human gut microbiome, metagenomic sequencing leads to high coverage of Bacteroidetes species which increases the likelihood that a phasevariable region can be detected with PhaseFinder (17). Though more than two thousand metagenomic samples were analyzed, no phase-variable ARGs were identified in other phyla, suggesting phase-variable ARGs might be rare in phyla other than Bacteroidetes.

This finding highlights the threat to human health that Bacteroidetes species pose as a reservoir for the dissemination of ARGs. The phylum Bacteroidetes is one of the most abundant in the human gut and Bacteroidetes species, especially those in genus Bacteroides, are regarded as a reservoir of antibiotic resistance genes (36). Moreover, members of Bacteroidetes species, such as Bacteroides fragilis, are considered opportunistic pathogens (37) and can be the causative agent of appendicitis and intra-abdominal abscesses $(38,39)$. Antibiotics have been used to treat such infections, but an increasing rate of antibiotic resistance has been noted in Bacteroidetes species (40-43). Phase-variable ARGs may contribute to the continued maintenance of clinically-relevant antibiotic resistance genes such as $c m e A B C$, erm $G$, and tet $Q$, which confer resistance to a widely-used antibiotics including macrolides, streptogramin and tetracycline $(42,44)$. Most species identified with phase-variable ARGs are considered commensals in the human gut. However, ARGs transfer from commensals to pathogens through horizontal transfer has been documented $(5,45,46)$. The transfer of phase-variable ARGs from commensals to pathogens via MGEs, such as the ICEs identified in this study, might 
promote resistance to a wide array of antibiotics in pathogenic species (47), posing a threat to public health in the future. Due to the fact that inter-phylum transfer of ICEs is rare (30), the spread of phase-variable ARGs might be limited within the phylum Bacteroidetes. As such, monitoring these Bacteroidetes species, especially the ICEs in these species, might be important in mitigating the threat of phase-variable ARGs to human health.

\section{MATERIALS AND METHODS}

\section{De novo assembly and gene annotation of metagenomic datasets}

We downloaded metagenomic sequencing data that consisted of 2227 human gut samples encompassing 7 studies (Table S1). Low-quality reads were removed and sequencing adapters were trimmed with trim_galore (v0.6.4; https://github.com/FelixKrueger/TrimGalore). The filtered data were mapped to the human genome (hg19) using bowtie2 (v2.3.5.1) (48) to filter human reads. The cleaned reads from each sample were assembled with SPAdes (v3.14.0) using the -meta option $(49,50)$, and sequences less than 500 bp were removed. Gene annotation was performed using Prokka (v1.14.5) (51) with the parameter --metagenome.

\section{Annotation of antibiotic resistance genes (ARGs)}

We searched the annotated sequences for known ARGs from the Comprehensive Antibiotic Resistance Database (CARD) (v3.0.7) (52) using Blastn (v2.10.0) (53). The BLAST results were filtered using the parameters: -perc_identity 80, -evalue 1e-10, and -culling_limit 1. Resistance Gene Identifier (RGI, v5.0.0) (52) was also used to predict known antibiotic resistance elements using the following parameters: rgi main --t contig -a BLAST -n 8 -d wgs --local. In addition, we also searched the sequences against the Resfams HMM Database (Core) (54) using hmmscan with the parameter: --cut_ga.

\section{Identification of phase-variable ARGs}

ARGs, along with upstream and downstream 20,000 flanking bases, were extracted to identify invertons using PhaseFinder (v1.0) (17). The default parameters of PhaseFinder were used. We filtered the results by removing the invertible DNA regions with $<2$ reads supporting the $R$ orientation from the paired-end method, and the $\mathrm{Pe}$ _ratio $<1 \%$. Furthermore, the invertible DNA regions containing or overlapping coding sequences (CDS) were removed. The motifs of inverted repeats and promoters were identified based on the previously reported motifs (17) and the logos were generated with WebLogo (version 2.8.2) (55). 


\section{Identification of host species and mobile genetic elements}

We identified the host species for the identified contigs using blastn by searching the non-MGE sequences against the NCBI non-redundant nucleotide (nt) database and the Unified Human Gastrointestinal Genome UHGG database (29) with an e-value <1-e10. We performed blastn to search for identical homologs (> 99\% identity) of the identified invertases and invertible regions against the 204,938 non-redundant genomes from the UHGG database. The identical homologs were grouped into corresponding classes of IP-ARG and the UHGG annotations for host species were examined to identify host species for IP-ARG.

To identify integrative and conjugative elements (ICEs) in different classes of IP-ARGs, we searched the ICEberg $(31,56)$ and ImmeDB $(30)$ databases using blastn with an e-value $<1$ e10. We used ConjScan $(57,58)$ via a Galaxy web server (https://galaxy.pasteur.fr) to annotate conjugative genes in ICEs.

\section{Genomic comparison and phylogenetic analysis}

The bacteria genomes and genome comparisons were visualized with the $\mathrm{R}$ package genoPlotR. All identified invertases were used to construct the phylogenetic tree for the classification of IP-ARGs. To understand the evolutionary history of IP-ARGs, the invertase genes regulating phase-variable ARGs identified in our assembled sequences and their homologs in the UHGG database, as well as those that have been previously reported(17) were used to construct the phylogenetic tree. Redundant invertase genes were filtered using cd-hit with the $99 \%$ identity threshold (59). Only the invertase genes regulating functionally characterized genes or operons were included in the tree. Multiple alignments of invertase gene nucleotide sequences were performed with MUSCLE (v3.8.31) (60). The alignment results were analyzed in FastTree (v2.1.10) (61) with default parameters to infer the phylogenetic trees. The phylogenetic trees were visualized using iTOL (https://itol.embl.de).

\section{References}

1. Alekshun MN, Levy SB. 2007. Molecular Mechanisms of Antibacterial Multidrug Resistance. Cell.

2. Sommer MOA, Dantas G, Church GM. 2009. Functional characterization of the antibiotic resistance reservoir in the human microflora. Science 325:1128-1131.

3. Kim S, Covington A, Pamer EG. 2017. The intestinal microbiota: Antibiotics, colonization 
resistance, and enteric pathogens. Immunol Rev 279:90-105.

4. Wallace MJ, Fishbein SRS, Dantas G. 2020. Antimicrobial resistance in enteric bacteria: current state and next-generation solutions. Gut Microbes 12:1799654.

5. Broaders E, Gahan CGM, Marchesi JR. 2013. Mobile genetic elements of the human gastrointestinal tract: potential for spread of antibiotic resistance genes. Gut Microbes 4:271-280.

6. Bilgin N, Claesens F, Pahverk H, Ehrenberg M. 1992. Kinetic properties of Escherichia coli ribosomes with altered forms of S12. J Mol Biol 224:1011-1027.

7. Andersson DI, Levin BR. 1999. The biological cost of antibiotic resistance. Current Opinion in Microbiology.

8. Hernando-Amado S, Sanz-García F, Blanco P, Martínez JL. 2017. Fitness costs associated with the acquisition of antibiotic resistance. Essays Biochem 61:37-48.

9. Andersson DI, Hughes D. 2010. Antibiotic resistance and its cost: is it possible to reverse resistance? Nat Rev Microbiol 8:260-271.

10. Sander P, Springer B, Prammananan T, Sturmfels A, Kappler M, Pletschette M, Böttger EC. 2002. Fitness Cost of Chromosomal Drug Resistance-Conferring Mutations.

Antimicrobial Agents and Chemotherapy.

11. Ramadhan AA. 2005. Survivability of vancomycin resistant enterococci and fitness cost of vancomycin resistance acquisition. Journal of Clinical Pathology.

12. Schrag SJ, Perrot V. 1996. Reducing antibiotic resistance. Nature 381:120-121.

13. Björkman J, Nagaev I, Berg OG, Hughes D, Andersson DI. 2000. Effects of environment on compensatory mutations to ameliorate costs of antibiotic resistance. Science 287:14791482.

14. Comas I, Borrell S, Roetzer A, Rose G, Malla B, Kato-Maeda M, Galagan J, Niemann S, Gagneux S. 2011. Whole-genome sequencing of rifampicin-resistant Mycobacterium tuberculosis strains identifies compensatory mutations in RNA polymerase genes. Nat Genet 44:106-110.

15. Enne VI, Bennett PM, Livermore DM, Hall LMC. 2004. Enhancement of host fitness by the 
sul2-coding plasmid p9123 in the absence of selective pressure. J Antimicrob Chemother 53:958-963.

16. Roberts AP, Mullany P. 2011. Tn916-like genetic elements: a diverse group of modular mobile elements conferring antibiotic resistance. FEMS Microbiol Rev 35:856-871.

17. Jiang $X$, Hall AB, Arthur TD, Plichta DR, Covington CT, Poyet M, Crothers J, Moses PL, Tolonen AC, Vlamakis H, Alm EJ, Xavier RJ. 2019. Invertible promoters mediate bacterial phase variation, antibiotic resistance, and host adaptation in the gut. Science 363:181-187.

18. Reyes Ruiz LM, Williams CL, Tamayo R. 2020. Enhancing bacterial survival through phenotypic heterogeneity. PLoS Pathog 16:e1008439.

19. Trzilova D, Tamayo R. 2021. Site-Specific Recombination - How Simple DNA Inversions Produce Complex Phenotypic Heterogeneity in Bacterial Populations. Trends Genet 37:5972.

20. Henderson IR, Owen P, Nataro JP. 1999. Molecular switches--the ON and OFF of bacterial phase variation. Mol Microbiol 33:919-932.

21. van der Woude MW. 2011. Phase variation: how to create and coordinate population diversity. Curr Opin Microbiol 14:205-211.

22. Villemur R, Déziel E. 2005. Phase variation and antigenic variation. The Dynamic Bacterial Genome.

23. Abraham JM, Freitag CS, Clements JR, Eisenstein BI. 1985. An invertible element of DNA controls phase variation of type 1 fimbriae of Escherichia coli. Proc Natl Acad Sci U S A 82:5724-5727.

24. Olsen PB, Klemm P. 1994. Localization of promoters in the fim gene cluster and the effect of H-NS on the transcription of fimB and fimE. FEMS Microbiol Lett 116:95-100.

25. Bonifield HR, Hughes KT. 2003. Flagellar phase variation in Salmonella enterica is mediated by a posttranscriptional control mechanism. J Bacteriol 185:3567-3574.

26. Coyne MJ, Chatzidaki-Livanis M, Paoletti LC, Comstock LE. 2008. Role of glycan synthesis in colonization of the mammalian gut by the bacterial symbiont Bacteroides fragilis. Proc Natl Acad Sci U S A 105:13099-13104. 
27. Krinos CM, Coyne MJ, Weinacht KG, Tzianabos AO, Kasper DL, Comstock LE. 2001. Extensive surface diversity of a commensal microorganism by multiple DNA inversions. Nature 414:555-558.

28. Sekulovic O, Garrett EM, Bourgeois J, Tamayo R, Shen A, Camilli A. 2018. Genome-wide detection of conservative site-specific recombination in bacteria. PLOS Genetics.

29. Almeida A, Nayfach S, Boland M, Strozzi F, Beracochea M, Shi ZJ, Pollard KS, Sakharova E, Parks DH, Hugenholtz P, Segata N, Kyrpides NC, Finn RD. 2020. A unified catalog of 204,938 reference genomes from the human gut microbiome. Nat Biotechnol https://doi.org/10.1038/s41587-020-0603-3.

30. Jiang X, Hall AB, Xavier RJ, Alm EJ. 2019. Comprehensive analysis of chromosomal mobile genetic elements in the gut microbiome reveals phylum-level niche-adaptive gene pools. PLoS One 14:e0223680.

31. Liu M, Li X, Xie Y, Bi D, Sun J, Li J, Tai C, Deng Z, Ou H-Y. 2019. ICEberg 2.0: an updated database of bacterial integrative and conjugative elements. Nucleic Acids Res 47:D660D665.

32. Chatzidaki-Livanis M, Coyne MJ, Comstock LE. 2009. A family of transcriptional antitermination factors necessary for synthesis of the capsular polysaccharides of Bacteroides fragilis. J Bacteriol 191:7288-7295.

33. Coyne MJ, Comstock LE. 2008. Niche-specific features of the intestinal bacteroidales. J Bacteriol 190:736-742.

34. Patrick S, Blakely GW, Houston S, Moore J, Abratt VR, Bertalan M, Cerdeño-Tárraga AM, Quail MA, Corton N, Corton C, Bignell A, Barron A, Clark L, Bentley SD, Parkhill J. 2010. Twenty-eight divergent polysaccharide loci specifying within- and amongst-strain capsule diversity in three strains of Bacteroides fragilis. Microbiology 156:3255-3269.

35. van der Woude MW, Bäumler AJ. 2004. Phase and antigenic variation in bacteria. Clin Microbiol Rev 17:581-611, table of contents.

36. Salyers A, Gupta A, Wang Y. 2004. Human intestinal bacteria as reservoirs for antibiotic resistance genes. Trends in Microbiology.

37. Wexler HM. 2007. Bacteroides: the good, the bad, and the nitty-gritty. Clin Microbiol Rev 
$20: 593-621$.

38. Bennion RS, Baron EJ, Thompson JE Jr, Downes J, Summanen P, Talan DA, Finegold SM. 1990. The bacteriology of gangrenous and perforated appendicitis--revisited. Ann Surg 211:165-171.

39. Jotwani R, Gupta U, Watanabe K, Ueno K. 1992. Pathogenicity of Bacteroides fragilis group in rat intra-abdominal abscesses. Microbiol Immunol 36:1041-1049.

40. Snydman DR, Jacobus NV, McDermott LA, Golan Y, Hecht DW, Goldstein EJC, Harrell L, Jenkins S, Newton D, Pierson C, Rihs JD, Yu VL, Venezia R, Finegold SM, Rosenblatt JE, Gorbach SL. 2010. Lessons learned from the anaerobe survey: historical perspective and review of the most recent data (2005-2007). Clin Infect Dis 50 Suppl 1:S26-33.

41. Nagy E, Urbán E, Nord CE, ESCMID Study Group on Antimicrobial Resistance in Anaerobic Bacteria. 2011. Antimicrobial susceptibility of Bacteroides fragilis group isolates in Europe: 20 years of experience. Clin Microbiol Infect 17:371-379.

42. Eitel Z, Sóki J, Urbán E, Nagy E, ESCMID Study Group on Anaerobic Infection. 2013. The prevalence of antibiotic resistance genes in Bacteroides fragilis group strains isolated in different European countries. Anaerobe 21:43-49.

43. Veloo ACM, Baas WH, Haan FJ, Coco J, Rossen JW. 2019. Prevalence of antimicrobial resistance genes in Bacteroides spp. and Prevotella spp. Dutch clinical isolates. Clin Microbiol Infect 25:1156.e9-1156.e13.

44. Niestępski S, Harnisz M, Korzeniewska E, Aguilera-Arreola MG, Contreras-Rodríguez A, Filipkowska Z, Osińska A. 2019. The emergence of antimicrobial resistance in environmental strains of the Bacteroides fragilis group. Environ Int 124:408-419.

45. Stecher B, Denzler R, Maier L, Bernet F, Sanders MJ, Pickard DJ, Barthel M, Westendorf AM, Krogfelt KA, Walker AW, Ackermann M, Dobrindt U, Thomson NR, Hardt W-D. 2012. Gut inflammation can boost horizontal gene transfer between pathogenic and commensal Enterobacteriaceae. Proc Natl Acad Sci U S A 109:1269-1274.

46. Thanh Duy P, Thi Nguyen TN, Vu Thuy D, Chung The H, Alcock F, Boinett C, Dan Thanh HN, Thanh Tuyen H, Thwaites GE, Rabaa MA, Baker S. 2020. Commensal Escherichia coli are a reservoir for the transfer of XDR plasmids into epidemic fluoroquinolone-resistant Shigella sonnei. Nat Microbiol 5:256-264. 
47. Botelho J, Schulenburg H. 2020. The Role of Integrative and Conjugative Elements in Antibiotic Resistance Evolution. Trends Microbiol 29:8-18.

48. Langmead B, Salzberg SL. 2012. Fast gapped-read alignment with Bowtie 2. Nat Methods 9:357-359.

49. Bankevich A, Nurk S, Antipov D, Gurevich AA, Dvorkin M, Kulikov AS, Lesin VM, Nikolenko SI, Pham S, Prjibelski AD, Pyshkin AV, Sirotkin AV, Vyahhi N, Tesler G, Alekseyev MA, Pevzner PA. 2012. SPAdes: a new genome assembly algorithm and its applications to single-cell sequencing. J Comput Biol 19:455-477.

50. Nurk S, Meleshko D, Korobeynikov A, Pevzner PA. 2017. metaSPAdes: a new versatile metagenomic assembler. Genome Res 27:824-834.

51. Seemann T. 2014. Prokka: rapid prokaryotic genome annotation. Bioinformatics 30:20682069.

52. Jia B, Raphenya AR, Alcock B, Waglechner N, Guo P, Tsang KK, Lago BA, Dave BM, Pereira S, Sharma AN, Doshi S, Courtot M, Lo R, Williams LE, Frye JG, Elsayegh T, Sardar D, Westman EL, Pawlowski AC, Johnson TA, Brinkman FSL, Wright GD, McArthur AG. 2017. CARD 2017: expansion and model-centric curation of the comprehensive antibiotic resistance database. Nucleic Acids Res 45:D566-D573.

53. Altschul SF, Gish W, Miller W, Myers EW, Lipman DJ. 1990. Basic local alignment search tool. J Mol Biol 215:403-410.

54. Gibson MK, Forsberg KJ, Dantas G. 2015. Improved annotation of antibiotic resistance determinants reveals microbial resistomes cluster by ecology. ISME J 9:207-216.

55. Crooks GE, Hon G, Chandonia J-M, Brenner SE. 2004. WebLogo: a sequence logo generator. Genome Res 14:1188-1190.

56. Bi D, Xu Z, Harrison EM, Tai C, Wei Y, He X, Jia S, Deng Z, Rajakumar K, Ou H-Y. 2012. ICEberg: a web-based resource for integrative and conjugative elements found in Bacteria. Nucleic Acids Res 40:D621-6.

57. Abby SS, Néron B, Ménager H, Touchon M, Rocha EPC. 2014. MacSyFinder: a program to mine genomes for molecular systems with an application to CRISPR-Cas systems. PLoS One 9:e110726. 
58. Abby SS, Cury J, Guglielmini J, Néron B, Touchon M, Rocha EPC. 2016. Identification of protein secretion systems in bacterial genomes. Scientific Reports.

59. Li W, Godzik A. 2006. Cd-hit: a fast program for clustering and comparing large sets of protein or nucleotide sequences. Bioinformatics 22:1658-1659.

60. Edgar RC. 2004. MUSCLE: a multiple sequence alignment method with reduced time and space complexity. BMC Bioinformatics 5:113.

61. Price MN, Dehal PS, Arkin AP. 2009. FastTree: computing large minimum evolution trees with profiles instead of a distance matrix. Mol Biol Evol 26:1641-1650.

\section{Acknowledgments}

We thank NIH's Biowulf cluster team. This work utilized the computational resources of the NIH HPC Biowulf cluster (http://hpc.nih.gov).

W.Y. and X.J. is supported by the Intramural Research Program of the NIH, National Library of Medicine. B.H. is supported by startup funding from the University of Maryland.

\section{Contributions}

X.J. conceived and designed the study. W.Y. performed the data analysis and wrote the draft manuscript. X.J. and A.B.H. reviewed and revised the manuscript. X.J. supervised the work. All authors read and approved the final manuscript.

\section{Competing interests}

The authors declare no competing interests. 
1 Table 1. Taxonomic and geographic distribution of the phase-variable ARGs.

\begin{tabular}{|c|c|c|c|c|c|}
\hline \multirow{2}{*}{$\begin{array}{l}\text { The class of identified } \\
\text { Integrase genes and } \\
\text { identical homologs } \\
\text { regulating ARGs } \\
\end{array}$} & \multicolumn{4}{|c|}{ Bacteria host $^{1}$} & \multirow{2}{*}{ Country } \\
\hline & Order & Family & Genus & Species & \\
\hline \multirow[t]{6}{*}{1} & Bacteroidales & Bacteroidaceae & Bacteroides & $\begin{array}{l}\text { Bacteroides cellulosilyticus, Bacteroides eggerthii, } \\
\text { Bacteroides fluxus, Bacteroides fragilis, Bacteroides } \\
\text { fragilis_A, Bacteroides intestinalis, Bacteroides ovatus, } \\
\text { Bacteroides stercoris, Bacteroides thetaiotaomicron, } \\
\text { Bacteroides uniformis, Bacteroides xylanisolvens, } \\
\text { Unclassified species }\end{array}$ & \multirow{6}{*}{$\begin{array}{l}\text { Austria, Canada, } \\
\text { China, Denmark, } \\
\text { Estonia, Finland, } \\
\text { France, Germany, } \\
\text { Israel, Japan, } \\
\text { Kazakhstan, } \\
\text { Netherlands, } \\
\text { Russia, Spain, } \\
\text { Sweden, } \\
\text { United Kingdom, } \\
\text { United States }\end{array}$} \\
\hline & & & Phocaeicola & $\begin{array}{l}\text { Phocaeicola coprocola, Phocaeicola coprophilus, Phocaeicola } \\
\text { dorei, Phocaeicola sp000432735, Phocaeicola vulgatus }\end{array}$ & \\
\hline & & & Prevotella & $\begin{array}{l}\text { Prevotella copri, Prevotella lascolaii, Prevotella sp000834015, } \\
\text { Prevotella sp001275135, Prevotella sp900313215, Prevotella } \\
\text { stercorea }\end{array}$ & \\
\hline & & Barnesiellaceae & Barnesiella & Barnesiella intestinihominis & \\
\hline & & Coprobacteraceae & Coprobacter & Coprobacter fastidiosus & \\
\hline & & Tannerellaceae & Parabacteroides & $\begin{array}{l}\text { Parabacteroides distasonis, Parabacteroides goldsteinii, } \\
\text { Parabacteroides merdae }\end{array}$ & \\
\hline \multirow[t]{3}{*}{2} & Bacteroidales & Bacteroidaceae & Bacteroides & $\begin{array}{l}\text { Bacteroides caccae, Bacteroides eggerthii, Bacteroides } \\
\text { fragilis, Bacteroides thetaiotaomicron, Bacteroides uniformis }\end{array}$ & \multirow{3}{*}{$\begin{array}{l}\text { China, Denmark, } \\
\text { Estonia, Israel, } \\
\text { Italy, } \\
\text { United Kingdom, } \\
\text { United States }\end{array}$} \\
\hline & & & Paraprevotella & Paraprevotella xylaniphila & \\
\hline & & & Phocaeicola & Phocaeicola dorei & \\
\hline \multirow[t]{2}{*}{3} & Bacteroidales & Bacteroidaceae & Phocaeicola & Phocaeicola dorei & Denmark \\
\hline & & UBA932 & RC9 & RC9 sp000434935 & \\
\hline
\end{tabular}

$2{ }^{1}$ Taxonomic information are annotated based on GTDB release95. 


\section{Figures}

4

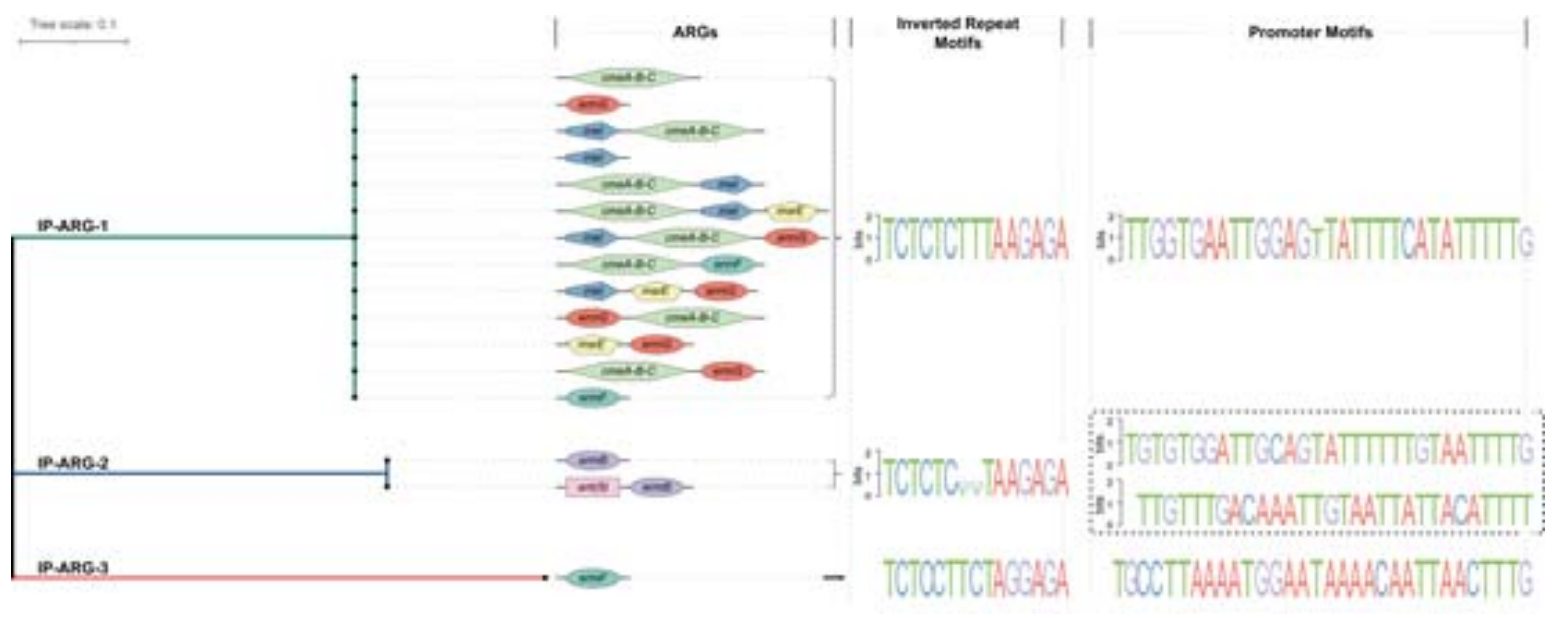

5 Figure 1: Phase-variable ARGs were classified into three classes (IP-ARG-1, IP-ARG-2, 6 and IP-ARG-3) based on the alignment of nucleotide sequences of invertase genes. The

7 ARGs and putative ARG organization patterns are shown for each class. Different ARGs are 8 shown in different shapes and colors. 


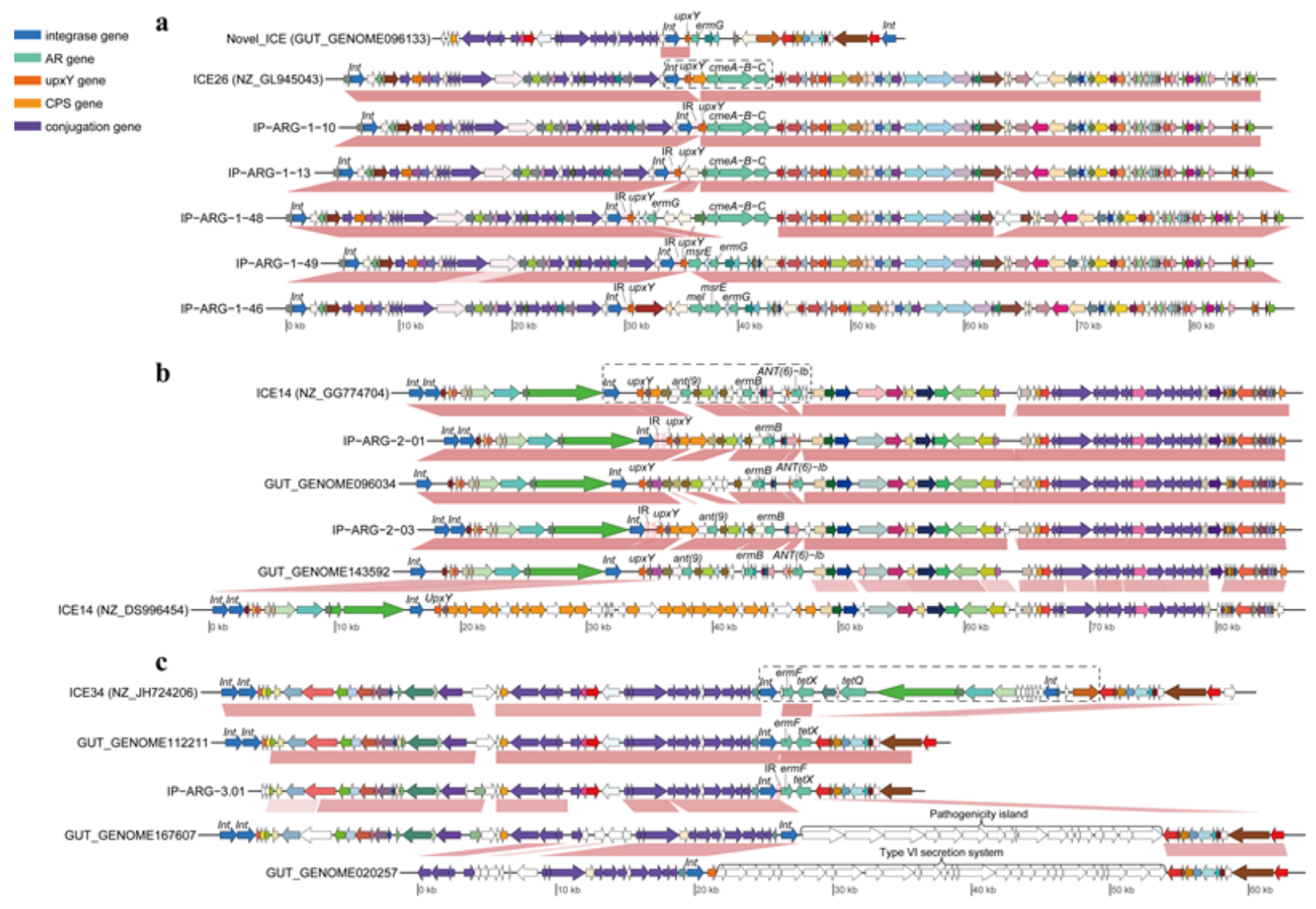

Figure 2: Genomic comparison and context analysis of the integrative and conjugative element (ICE) variants carrying IP-ARG-1 (a), IP-ARG-2 (b), or IP-ARG-3 (c). The regions located adjacent to the invertible promoters (black dotted boxes) were found to be highly variable across element variants within each ICE. ICE26, ICE14, and ICE34 are ICE accession numbers from ImmeDB. The NCBI genome accession numbers are shown in the parentheses after the ICE accession numbers. The sequence labels that start with GUT_GENOME are genome accession numbers of the UHGG database. Orthologous genes are plotted with the

17 same color and are linked by pink connections. Invertase and antibiotic resistance genes are colored blue and light green, respectively. Genes involved in conjugation and capsular polysaccharide biosynthesis are colored purple and orange, respectively. The genes that do not have orthologs are white. Invertase, $u p x Y$, and antibiotic resistance genes as well as inverted 21 repeats are labeled. AR, CPS, INV, and IR denote antibiotic resistance, capsular 22 polysaccharides, invertase, and inverted repeats, respectively. 


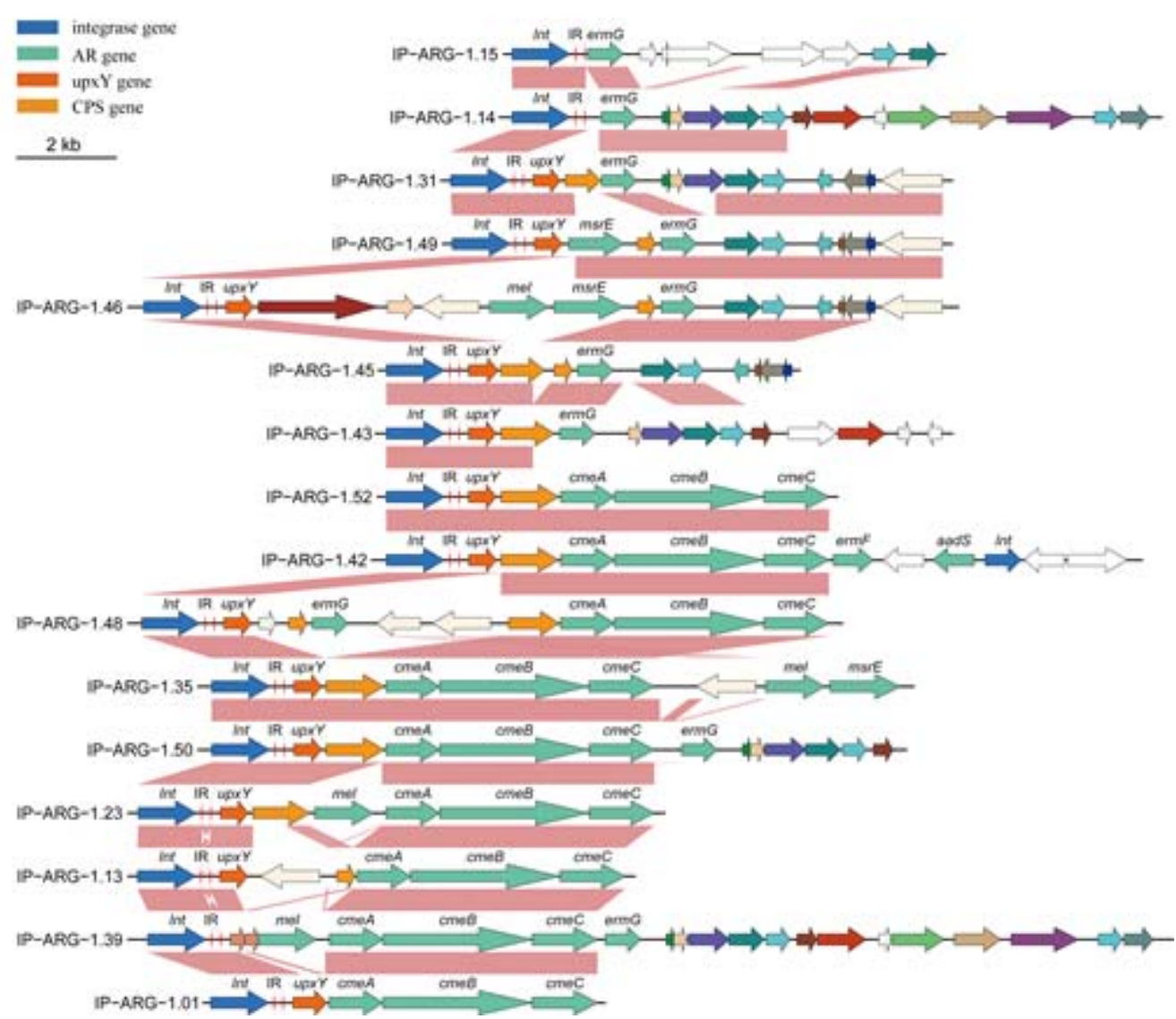

24 Figure 3: Comparisons of highly variable regions carrying IP-ARG-1 demonstrate the 25 degeneration of the upx $Y$ gene. Different degeneration statuses of the upxY gene were: 26 partially degenerated (IP-ARG-1-39), completely degenerated (IP-ARG-1-14 and IP-ARG-1-15), 27 fusion gene (IP-ARG-1-01), and intact gene (the remainder). Orthologous genes are plotted with 28 the same color and are linked by pink connections. Invertase genes and antibiotic resistance 29 genes are colored blue and light green, respectively. Genes involved in conjugation and 30 capsular polysaccharide biosynthesis are colored purple and orange, respectively. The genes 31 that do not have orthologs are white. Invertase, $u p x Y$, and antibiotic resistance genes as well as 32 inverted repeats are labeled. AR, CPS, INV, and IR denote antibiotic resistance, capsular 33 polysaccharides, invertase, and inverted repeats, respectively. 


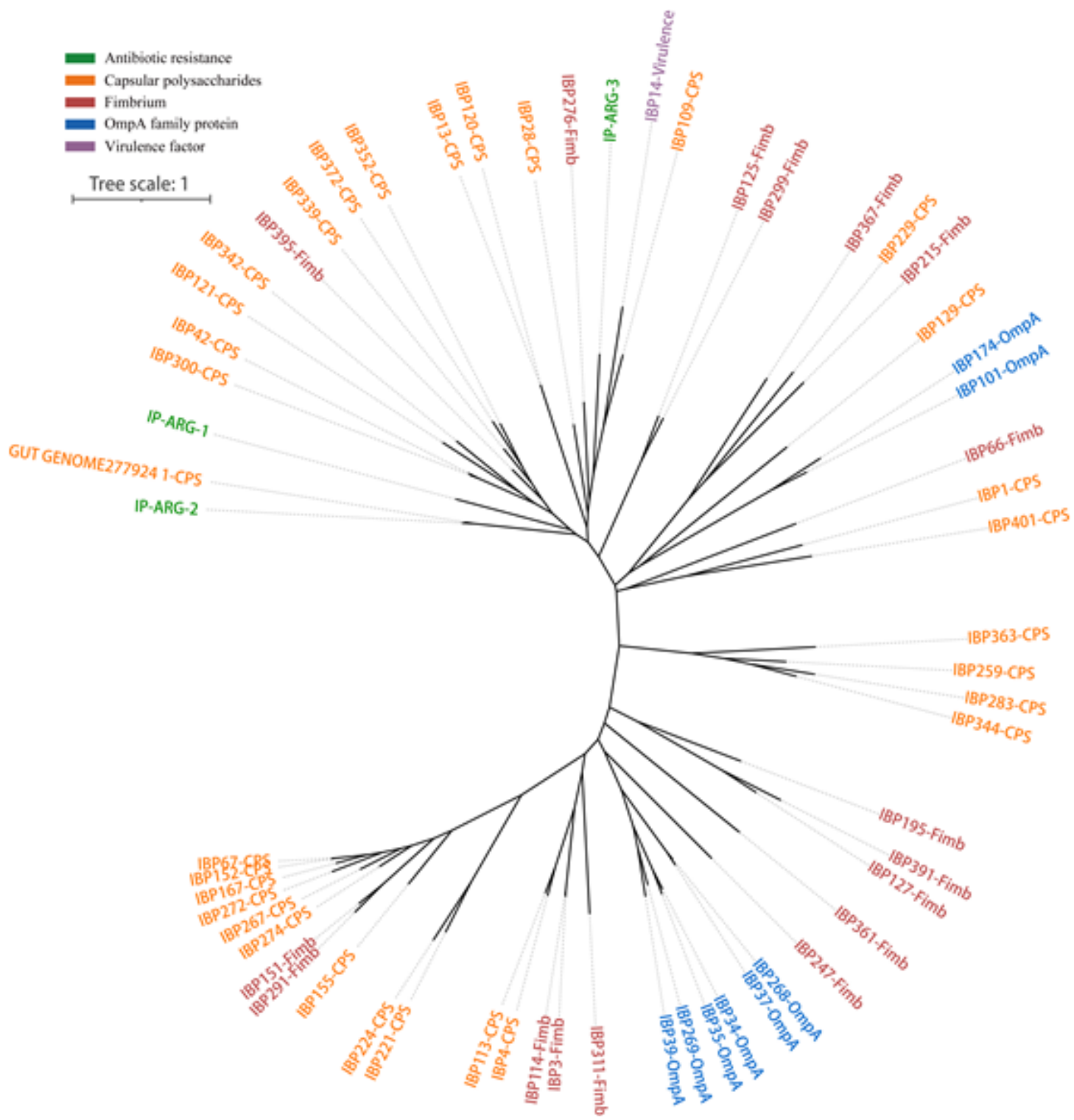

35 Figure 4: The evolutionary events that led to the emergence of phase-variable ARGs may 36 have occurred independently at least three times as the result of convergent evolution.

37 The phylogenetic tree was inferred based on the alignment of nucleotide sequences of invertase 38 genes. The invertase genes include those mediating phase-variable ARGs identified in our 39 assembled sequences and their homologs in the UHGG database as well as those that have 40 been previously reported(17). IBP denotes the invertible DNA region in phylum Bacteroidetes, 41 which is designated in the previous study(17). The labels that start with GUT_GENOME are 42 genome accessions in the UHGG database. Labels are colored based on the functional 43 annotation of the loci regulated by the invertase genes and invertible regions. 


\section{Supplementary Information}

\section{Supplementary Data:}

46 Supplementary Data 1: Nucleotide sequences of the identified contigs carrying phase-variable 47 ARGs.

\section{Supplementary Tables:}

49 Table S1: Human gut metagenomic samples analyzed in this study.

50 Table S2: Sequences containing phase-variable antibiotic resistance genes.

51 Table S3: UHGG genomes carrying the homologs of the identified invertase genes mediating 52 phase-variable ARGs. 
IP-ARG-1

IP-ARG-2

ermB -

ant(9) ermB

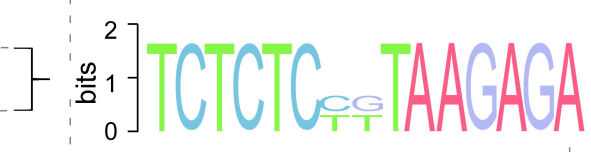

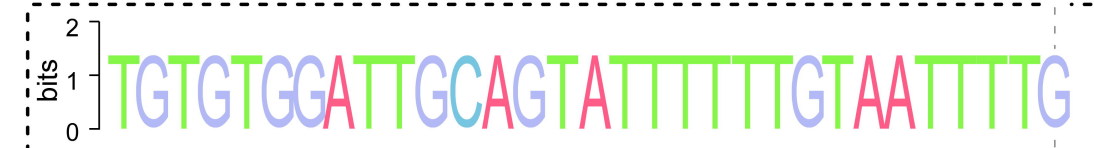

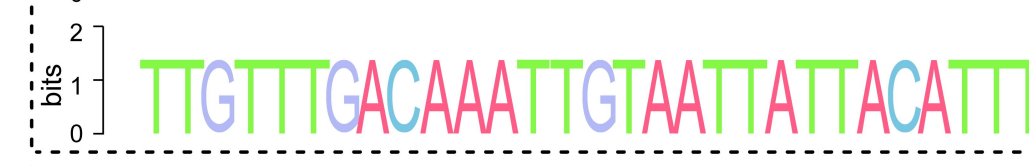

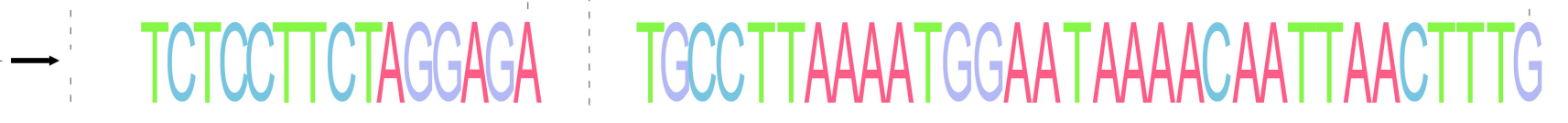




\section{invertase gene}

AR gene

upxY gene

CPS gene

$2 \mathrm{~kb}$
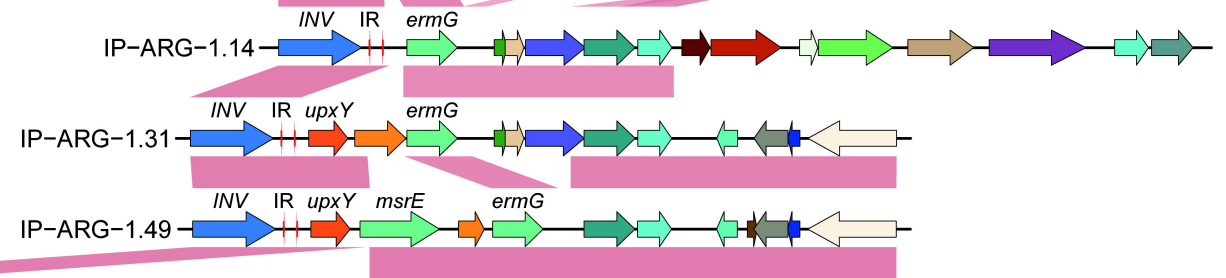

IP-ARG-1.46 INV IR upxY

rel

msrE ermG
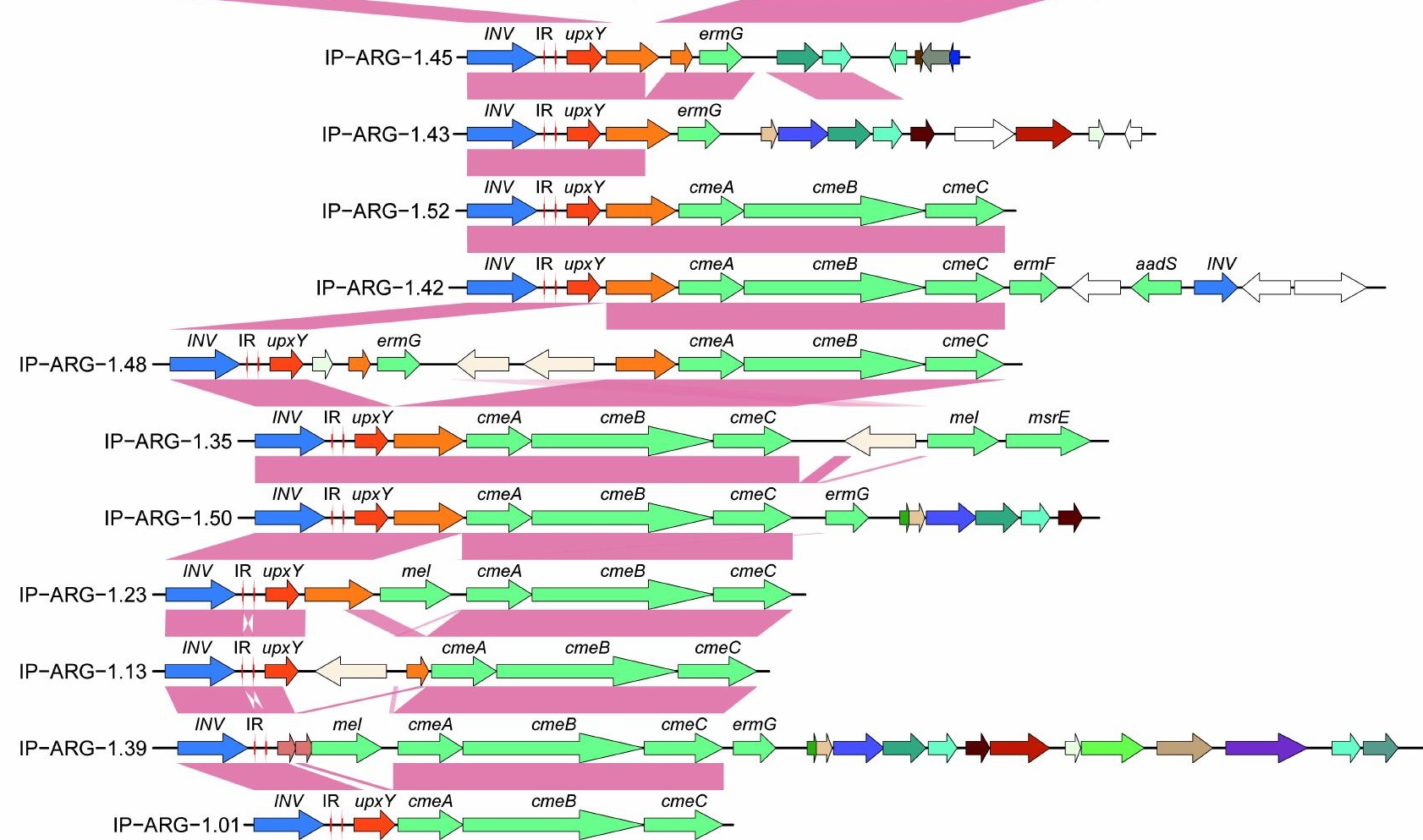
Antibiotic resistance

Capsular polysaccharides

Fimbrium

OmpA family protein

Virulence factor

Tree scale: 1

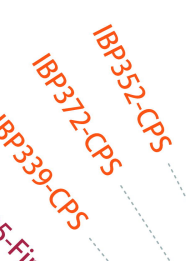

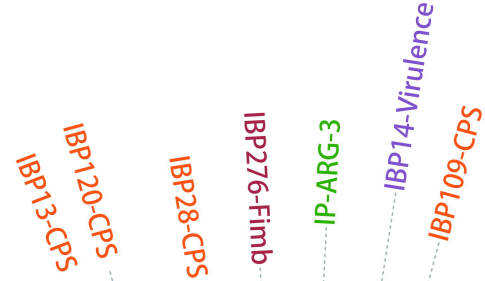
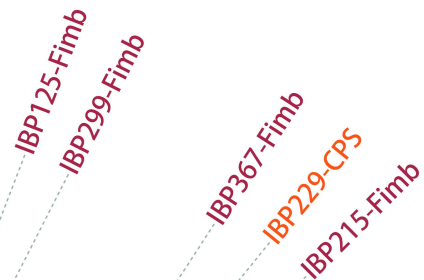

${ }_{1 B B^{3}} 0^{-} \mathrm{CP}$
${ }_{1 B D^{4}}$

IP-ARG-1

GUT GENOME277924 1-CPS

IP-ARG-2

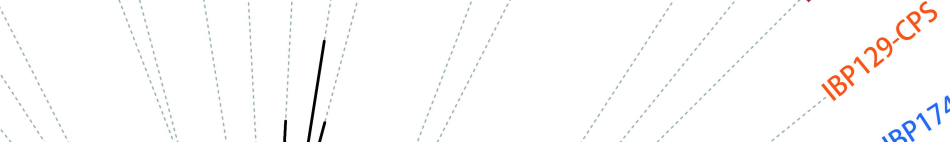

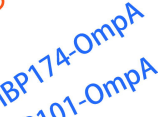

${ }_{B P 101}-0 m p$

1BP66-Fimb

IBP1-CPS

IBP401-CPS

IBP363-CPS

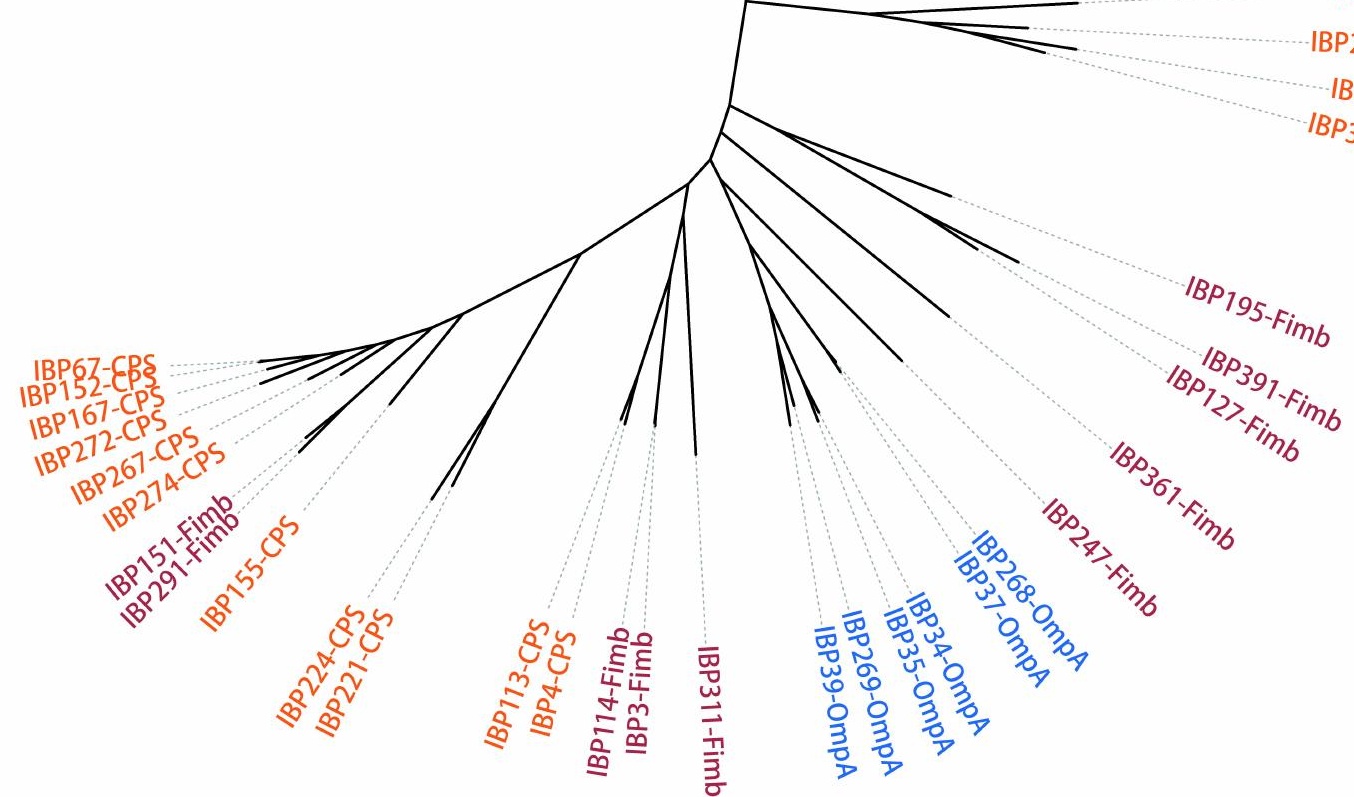

BP259-CPS

IBP283-CPS

344-CPS 